\title{
Vitamin E ameliorates iodine-induced cytotoxicity in thyroid
}

\author{
Jiashu Yu ${ }^{1,3}$, Zhongyan Shan'2,*, Wei Chong ${ }^{1,3}$, Jinyuan Mao ${ }^{1,3}$, Yuxiu Geng ${ }^{1,3}$, Caixia Zhang ${ }^{1,3}$, \\ Qian Xing ${ }^{1,3}$, Weiwei Wang ${ }^{1,2}$, Ningna $\mathrm{Li}^{1,3}$, Chenling Fan ${ }^{1,3}$, Hong Wang ${ }^{1,3}$, Hongmei Zhang ${ }^{1,3}$ \\ and Weiping Teng ${ }^{1,3, *}$ \\ ${ }^{1}$ The Endocrine Institute of China Medical University, Shenyang 110001, People's Republic of China \\ ${ }^{2}$ Department of Endocrinology and Metabolism, The First Hospital of China Medical University, Shenyang 110001, People's Republic of China \\ ${ }^{3}$ The Liaoning Provincial Key Laboratory of Endocrine Diseases, Shenyang 110001, People's Republic of China \\ (Correspondence should be addressed to W Teng at The Liaoning Provincial Key Laboratory of Endocrine Diseases, The Endocrine Institute of China Medical \\ University; Email: twpendocrine@yahoo.com.cn; Z Shan at Department of Endocrinology and Metabolism, The First Hospital of China Medical University; \\ Email: shanzhongyan@medmail.com.cn) \\ *(W Teng and Z Shan contributed equally to this work)
}

\begin{abstract}
Acute and excessive iodine supplementation leads to iodineinduced thyroid cytotoxicity. Excessive oxidative stress has been suggested to be one of the underlying mechanisms in the development of thyroid cytotoxicity. The aim of this study was to investigate whether vitamin $\mathrm{E}(\mathrm{VE})$, an important antioxidant, could ameliorate iodine-induced thyroid cytotoxicity. A goiter was induced in rats by feeding a low-iodine (LI) diet for 12 weeks. Involution of hyperplasia was obtained by administering a twofold physiological dose of iodine in feeding water with/without the supplementation of 25-, $50-$, or 100-fold physiological dose of VE in the LI diet for 4 weeks. In iodine-supplemented rats, thyroid epithelial cell ultrastructure injuries remained and were more severe. Relative weights of iodine-induced involuting glands were
\end{abstract}

significantly reduced compared with the goiter, but still higher than control. Immunohistochemistry indicated that the expression of 4-hydroxynonenal, 8-hydroxyguanine, peroxiredoxin 5, and CD68 in thyroid increased $(P<0 \cdot 01)$, whereas thioredoxin reductase 1 decreased $(P<0 \cdot 01)$. VE supplementation attenuated thyroid cytotoxicity induced by iodine. A 50-fold VE dose was optimal in attenuating twofold iodine-induced thyroid cytotoxicity. However, VE supplementation did not reduce the weight or relative weight of the iodine-induced involuting gland. These results show that excess iodine leads to thyroid damage and VE supplementation can partly ameliorate iodine-induced thyroid cytotoxicity.

Journal of Endocrinology (2011) 209, 299-306

\section{Introduction}

Thyrocytes constantly produce moderate amounts of reactive oxygen species (ROS), which are physiologically required for thyroid hormone synthesis (Denef et al. 1996, Song et al. 2007). Nevertheless, when ROS are over-produced, they may become toxic and damage cell structures and macromolecules including lipids, proteins, and nucleic acids. 4-Hydroxynonenal (4-HNE) is a toxic product resulting from lipid peroxidation and 8-hydroxyguanine (8-OHdG) and is a maker for DNA and RNA damage. A toxic effect of iodide given to iodine-deficient laboratory animals was first noted by Follis (1959). Similar studies have shown that 4-HNE is increased in goitrous and in iodine-induced involuting glands (iodine replenishment for 3 days, acute effects), indicating that oxidative stress (OS) is greatly enhanced in these conditions (Poncin et al. 2008). Increased OS is not necessarily lethal to goitrous cells but is associated with cellular destruction and inflammation in iodine-induced thyroid involution (Mutaku et al. 2002, Poncin et al. 2008).
It is therefore crucial for thyrocytes to develop protective mechanisms that limit the toxicity of endogenous and exogenous ROS. Fortunately, several antioxidants including superoxide dismutases, catalase, glutathione peroxidases (GPxs), peroxiredoxins (PRDXs), thioredoxin reductase (TrxR), and vitamin $\mathrm{E}$ (VE) are constitutively expressed in the thyroid (Mutaku et al. 2002, Gerard et al. 2005, Kohrle et al. 2005, Schweizer et al. 2008). Without these antioxidants, it would not be possible for these cells to remain viable and function properly. PRDX5 is a thioredoxin peroxidase that reduces or converts $\mathrm{H}_{2} \mathrm{O}_{2}$ into $\mathrm{H}_{2} \mathrm{O}$ and regulates intracellular levels of peroxides and peroxynitrites (Dubuisson et al. 2004, Banmeyer et al. 2005). PRDX5 is expressed in the cytosol of control thyrocytes as described previously in humans and rats (Gerard et al. 2005, Poncin et al. 2008, 2010). During their catalytic cycle, PRDXs (except PRDX6) are reduced by thioredoxins, which is reduced by TrxR (Lillig \& Holmgren 2007). Thioredoxin-TrxR pairs are ubiquitously expressed in the thyroid. Previous data from our laboratory indicate that iodine supplementation, to a level of 
more-than-adequate, in a region in which iodine intake was mildly deficient may accelerate the development of subclinical hypothyroidism to overt hypothyroidism (Teng et al. 2006). The iodine containing drug amiodarone has been found to induce thyrotoxicosis in geographical areas with iodine deficiency (Martino et al. 1984, Stanbury et al. 1998). Animal experiments indicate that chronic (iodine replenishment for several weeks) and adequate, more-than-adequate, or excessive iodine supplementation alone to iodine-deficient thyroids cannot cure iodine deficiency-induced injuries; on the contrary, excessive iodine supplement exacerbates thyroid damage (Chong et al. 2005, 2006a,b). Furthermore, antioxidant protection of the thyroid may be impaired by excessive iodine supplementation (Zhang et al. 2006). These findings suggest that excessive OS is one of the underlying mechanisms in the development of iodine-induced thyroid cytotoxicity. VE is classified as an antioxidant due to its ability to scavenge free radicals and terminate lipid oxidative chain reactions. VE has been demonstrated to protect against human diabetes mellitus with the $\mathrm{Hp} 2-2$ phenotype, idiopathic retinal periphlebitis, thalassemia, cerebral ische$\mathrm{mia} /$ reperfusion injury in irradiated rats, and steroid-induced osteonecrosis in rabbits (Milman et al. 2008, Abd-El-Fattah et al. 2010, Fibach \& Rachmilewitz 2010, Mikami et al. 2010, Saxena et al. 2010). VE, which is also very active in the antioxidative protection of thyrocyte membranes, is as concentrated in the thyroid as in the liver in control rats and is increased twofold in goiters (Mutaku et al. 1998). This indicates that VE is highly increased in the thyroid in cases of enhanced OS.

Unlike other experiments, in this study we used a lowiodine (LI) diet, without propylthiouracil (PTU) or perchlorate, to initiate goiter formation and then long-term small dose of iodine was administered (iodine replenishment for 4 weeks, chronic effects) to induce goiter involution (Chong et al. 2005, 2006a,b, Zhang et al. 2006). VE is a wellestablished antioxidant; however, it has never been reported to ameliorate thyroid injury induced by small doses of iodine. The purpose of this study was twofold: 1) to investigate OS and antioxidants expression, as well as ultrastructure changes of thyrocytes in a rat model of goiter formation and iodineinduced involution and 2) to examine the potential of VE protection on iodine-induced thyroid cytotoxicity.

\section{Materials and Methods}

\section{Animals and experiment protocol}

Hyperplastic goiter was induced in 4-week-old female Wistar rats (SLAC Laboratory Animal, Shanghai, China) by feeding a LI diet (dose of iodine in the LI diet is $60 \mu \mathrm{g} / \mathrm{kg}$ ) (Institute of Endocrinology, China Medical University, Shenyang, China) and demineralized water for 12 weeks. The weight of the rats
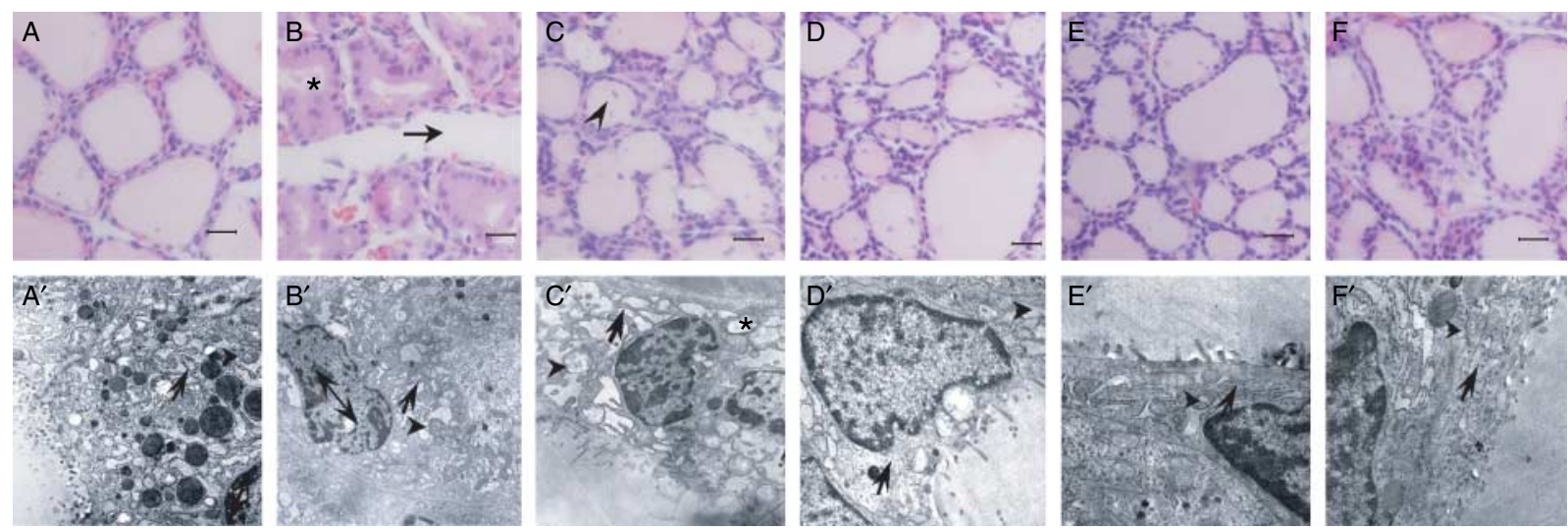

Figure 1 Thyroid morphology is an experimental model of goiter formation and excess iodine-induced involution supplemented with/without VE (A-F). (A) Thyroids from control rats had cubical epithelial cells and follicular lumina were filled with colloid. (B) Goitrous thyroids had markedly enlarged blood vessels (arrow), hypertrophic epithelial cells, and narrow follicular lumina $(*)$. (C) Thyroids of $2 \mathrm{l}$-induced involution demonstrated constricted blood vessels, widened follicular lumina, and some necrotic cells in lumina (arrowhead). (D-F) Thyroids with 25VE, 50VE, and 100VE supplementation on 2 l-induced involution rats showed regular follicles with colloid filled in their lumina. There was less cellular debris and no necrotic cells in the follicular lumina. Scale bar $=20 \mu \mathrm{m}$. Thyroid ultrastructure in the experimental model of goiter formation and 2 I-induced involution supplemented with/without $V E\left(A^{\prime}-F^{\prime}\right)$. $\left(A^{\prime}\right)$ Control thyroids $(\times 6400)$. Nuclei (two-way arrow) were round or ellipse. Endoplasmic reticulum (arrow) was deplanate. There were many ribosomes, mitochondria (arrowhead), colloid droplets, and lysosomes in cytoplasm. Plenty of microvilli were visible in the plasmalemma. $\left(\mathrm{B}^{\prime}\right)$ Thyroids of LI-induced goiter $(\times 2500)$ showed irregular nuclei (two-way arrow) and many incisures. Endoplasmic reticulum (arrow) was hypertrophied with very dilated cisternae and degranulated ribosomes. Mitochondria (arrowhead) had swelling and follicular lumina were not present. $\left(C^{\prime}\right)$ Thyroids from 2 l-induced involution rats $(\times 5000)$ demonstrated that endoplasmic reticulum cisternae (arrow) were much more dilated than goitrous thyroid. Some structures ruptured and were visible as unconnected vacuoles and large cavitation bubbles. Mitochondrial (arrowhead) swelling was increased, and mitochondrial cristae were blurred or even vacuolated $(*)$, with giant mitochondria presented. In three VE-supplemented thyroid involution groups (LI-2I+VE:D', LI-2I+25VE, X6000; E', LI-2I +50VE, X8000; F', LI-2I+ $100 \mathrm{VE}, \times 8000)$ mitochondria (arrowhead) were swollen and endoplasmic reticulum (arrow) was dilated and ameliorated. 
was $\sim 80 \mathrm{~g}$ at the onset of the study. Involution of hyperplasia was obtained by feeding water with a twofold physiological dose of iodine (a twofold iodine (2I) dose in water is $470 \cdot 7 \mu \mathrm{g} / \mathrm{l}$, and rats drank $\sim 20 \mathrm{ml}$ water/day) and LI diet with/without 25-, 50-, or 100-fold the physiological dosage of $\mathrm{VE}(25 \mathrm{VE}, 1875 \mathrm{IU} / \mathrm{kg}$; $50 \mathrm{VE}, 3750 \mathrm{IU} / \mathrm{kg}$; or $100 \mathrm{VE}$, $7500 \mathrm{IU} / \mathrm{kg}$ ) for 4 weeks ( $n=8$ per group). The VE dosages were based on previous reports by Many et al. (1991). To validate the experimental model and verify its reproducibility compared with previously published studies (Chong et al. 2005, 2006a,b, Zhang et al. 2006), the morphology of goitrous thyroids was examined as shown in Fig. 1B. The control group received a LI diet and water with normal iodine. Animals were killed using chloral hydrate. Rats were maintained in accordance with the principles of laboratory animal welfare. They were housed four per cage in a temperature- and lightcontrolled room and had free access to water and their respective diets. Each experimental setting was repeated twice. All procedures were approved by the local ethics committee.

\section{Weight and relative thyroid weight}

In total, eight animals per group were weighed prior to anesthesia. Thyroids were dissected, washed in cold physiological saline, blotted with filter paper, and weighed. The relative thyroid weight is calculated as the thyroid weight normalized to $100 \mathrm{~g}$ body weight.

\section{Electron microscope studies}

The right lobe of the thyroids was rapidly excised $1 \mathrm{~mm}^{3}$ and immersed in a $2 \cdot 5 \%$ glutaraldehyde solution at $4{ }^{\circ} \mathrm{C}$. After fixation, thyroid fragments were rinsed three times in cacodylate buffer, postfixed for $2 \mathrm{~h}$ in $1 \%$ osmium tetroxide, dehydrated in 50 and 70\% alcohol solutions, then 80 and $90 \%$ acetone solutions each for $30 \mathrm{~min}$, and finally in 100\% acetone for three times and for $10 \mathrm{~min}$. Thyroid fragments were embedded in Epon812 and aggregated in incubation at $35^{\circ} \mathrm{C}, 45^{\circ} \mathrm{C}$, and $60^{\circ} \mathrm{C}$ for $24 \mathrm{~h}$ at each temperature. Superthin sections $(0.05 \mu \mathrm{m})$ were cut (LKB-V, Bromma, Sweden) and stained with uranyl acetate and lead citrate and examined using a transmission electron microscope (H-600; Hitachi).

\section{Immunohistochemistry}

The left lobes were fixed in $4 \%$ paraformaldehyde and embedded in paraffin. Sections $(5 \mu \mathrm{m})$ were stained with hematoxylin-eosin or used for immunohistochemistry (IHC). CD68, 4-HNE, 8-OHdG, TrxR-1, and PRDX5 immunostains were performed on $5 \mu \mathrm{m}$-thick paraffin sections on Super Frost glass slides (Hehyglass, Haimen, China). Sections were dewaxed and rehydrated and treated for endogenous peroxidase with $3 \% \mathrm{H}_{2} \mathrm{O}_{2}$ for $10 \mathrm{~min}$. Except for PRDX5 detection, tissue sections were pretreated in a microwave oven in citrate buffer ( $\mathrm{pH} \mathrm{6.6)} \mathrm{for} 3 \mathrm{~min}$ at high power. All sections were washed with PBS and thereafter incubated with blocking sera for $30 \mathrm{~min}$ in a $37^{\circ} \mathrm{C}$ water bath. Sections were incubated with the primary antibodies (CD68, sc-70760, 1:50; 4-HNE, sc-130083, 1:50; 8-OHdG, sc-66036, 1:50; TrxR-1, sc-28321, 1:50; PRDX5, or sc-133072, 1:50) at $4{ }^{\circ} \mathrm{C}$ overnight. All antibodies were purchased from Santa Cruz Biotechnology (Santa Cruz, CA, USA). The binding of antibodies was detected using biotinylated secondary antibodies (Maixin, Fuzhou, China) for $30 \mathrm{~min}$ in a $37^{\circ} \mathrm{C}$ water bath, followed by an avidin-biotin-peroxidase complex (Maixin) for $30 \mathrm{~min}$ in a $37^{\circ} \mathrm{C}$ water bath. Peroxidase activity was visualized by $3,3^{\prime}$ diaminobenzidine tetrahydrochloride (Sigma). Sections were counter-stained with Mayer's hematoxylin, rinsed, and mounted in neutral gum (China National Medicines Corporation Ltd, Shanghai, China). To verify binding specificity, some sections were incubated with the secondary antibodies alone as there are no primary antibody controls. CD68-positive cells (monocytes and macrophages) were counted and reported as numbers per visual field. The expression of other proteins was determined by image information object definition (IOD) values analyzed by Image-Pro Plus 5.0 (Media Cybernetics, Silver Spring, MD, USA).

\section{Statistical analysis}

Data were analyzed using SPSS 17.0 software (Chicago, IL, USA) and expressed as mean \pm s.D. A two-sample $t$-test was used for comparison between two groups, and ANOVA was used for multi-group comparison. $P<0 \cdot 05$ was considered significant.

\section{Results}

Histomorphology of thyroid gland before and after iodine-induced involution

After 16 weeks of goitrogenic treatment, weight and the relative weight of the thyroids were significantly increased (Fig. 2A and B). The thyroid gland was hypervascularized and mainly consisted of hypertrophied follicular cells, whereas colloid had almost disappeared (Fig. 1B). Lysosomes were present and appeared normal. However, the endoplasmic
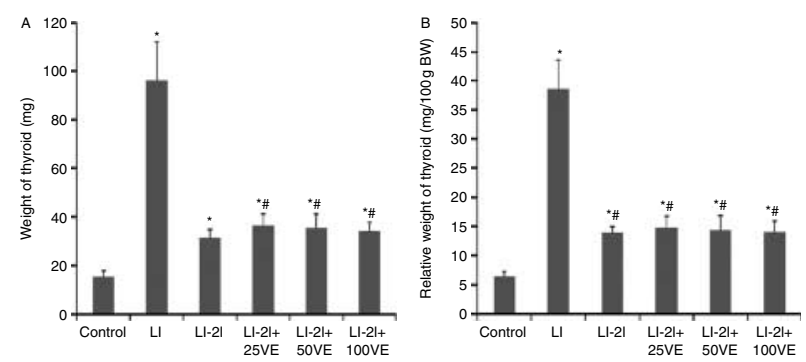

Figure 2 (A) Weight of thyroid (mg) (16 weeks). (B) Relative weight of thyroid (mg/100 g body weight) (16 weeks) $* P<0 \cdot 01$, compared with control; ${ }^{\sharp} P<0 \cdot 01$, compared with $\mathrm{LI}(n=8)$. 
reticulums (ERs) were hypertrophied with dilated cisternae and mitochondria were swollen. Microvilli were less developed than that in follicular cells of control thyroids. Nuclei were irregular (Fig. 1B' ${ }^{\prime}$ ). After 4 weeks of a twofold physiological dose of iodine supplementation, the weight and the relative weight of thyroid decreased; however, they were still higher than the control (Fig. 2A and B). Follicular lumina were enlarged and contained more colloid. Blood capillaries were constricted with scattered dead or fragmented cells in the follicular lumina. The interstitium was infiltrated with inflammatory cells (Fig. 1C). ER cisternae were much more dilated than that seen in the goitrous thyroid. Ruptured structures were present and formed unconnected vacuoles and large cavitation bubbles. There was an increase in swollen mitochondria, mitochondrial cristae were blurred or vacuolated, and giant mitochondria were present (Fig. 1C'). When VE was administered, the follicles and colloids were restored, and cell filtrations decreased (Fig. 1D-F). VE also ameliorated thyroid ultrastructure damage (Fig. $1 \mathrm{D}^{\prime}-\mathrm{F}^{\prime}$ ). However, VE-treatment had no effect on the weight and the relative weight of glands, which remained higher than control (Fig. 2A and B).

\section{OS and inflammation}

4-HNE, a toxic product of lipid peroxidation, was used as a marker for the OS. Weak constitutive 4-HNE immunostaining was detected in control thyroids (Fig. 3B), which was occasionally expressed in the plasma membrane and cytoplasm. 4-HNE expression was weaker in goiters but stronger in iodine-induced involutions $(P<0 \cdot 01$; Fig. $3 \mathrm{C}$ and D). The expression was evident in most of the plasma membrane and cytoplasm in iodine-induced involuting glands. The 4-HNE expression was significantly weaker in the 25- and 50-fold VE-treated rats $(P<0 \cdot 05)$ but not in 100-fold VE-treated rats (Fig. 3E-G).

$8-\mathrm{OHdG}$ is a marker of RNA and DNA oxidative damage. IHC revealed that $8-\mathrm{OHdG}$ was expressed in perinuclei cytoplasm in control thyroids (not shown). Its expression was diffuse in cytoplasm of goitrous thyroids (not shown). In iodine-treated animals, the staining was significantly more pronounced and more diffuse throughout the cytoplasm as well as some nuclei and interstitium (not shown). However, in three VE supplement groups, the 8-OHdG staining was significantly suppressed, especially in those groups receiving 50- and 100-fold levels of physiological VE $(P<0 \cdot 01)$ (not shown).

An antibody to CD68 was used to identify monocyte and macrophage infiltration. There were very few CD68-positive cells in control thyroids (Fig. 4B). In contrast, many positive cells were found in the thyroids of goitrous $(P<0 \cdot 01$ versus control) and iodine-treated goitrous rats and were particularly higher in the latter group $(P<0 \cdot 01$ versus LI; Fig. $4 \mathrm{C}$ and D). They were mostly localized in the interstitium, but also in capillaries, vascular lumina, and arterial walls. CD68-positive cells were also detected in VE supplement rats but to a significantly lower degree compared with $\mathrm{LI}+2 \mathrm{I}$ group $(P<0 \cdot 01$ for 25VE, 50VE, and 100VE groups) (Fig. 4E-G).
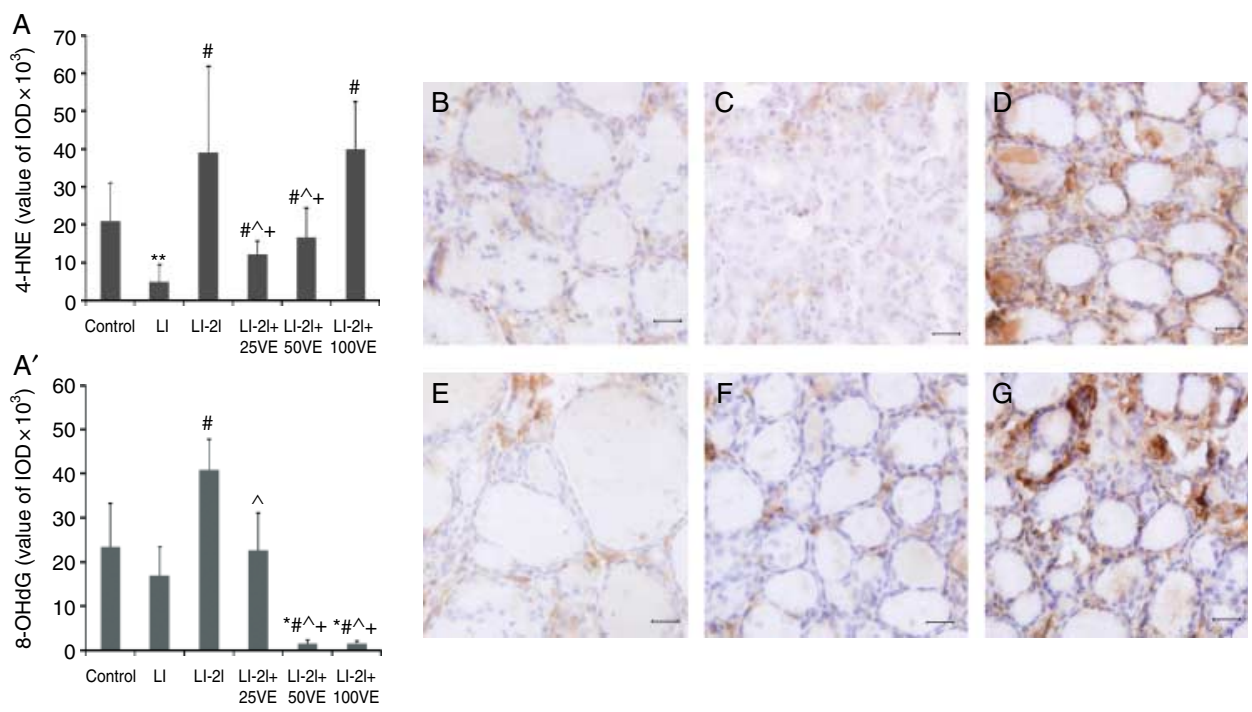

Figure 3 (A) IOD value of $4-\mathrm{HNE}, * * P<0 \cdot 01$, compared with control; ${ }^{*} P<0 \cdot 01$, compared with $\mathrm{LI}$; ${ }^{\wedge} P<0 \cdot 05$, compared with $\mathrm{LI}-2 \mathrm{I}$; $+P<0 \cdot 01$, compared with $\mathrm{LI}-2 \mathrm{I}+100 \mathrm{VE}(n=8)$. $\left(\mathrm{A}^{\prime}\right)$ IOD value of 8 -OHdG. ${ }^{*} P<0 \cdot 01$, compared with control; ${ }^{*} P<0 \cdot 01$, compared with $\mathrm{LI} ;{ }^{\wedge} P<0 \cdot 01$, compared with $\mathrm{LI}-2 \mathrm{I} ;{ }^{+} P<0 \cdot 01$, compared with $\mathrm{LI}-2 \mathrm{I}+25 \mathrm{VE}(n=8)$. (B-G) Immunohistochemical detection of $4-\mathrm{HNE}$. (B) In control thyroids, 4-HNE immunostaining was weakly expressed in plasma membrane and cytoplasm. (C) In LI-induced goiter thyroids, 4-HNE expression was even weaker. (D) 4-HNE expression was stronger with most plasma membrane and cytoplasm labeled in $2 \mathrm{I}$-induced involuting thyroids. (E and F) 25- and 50-fold VE-suppressed 4-HNE expression in $2 \mathrm{I}$-induced involuting thyroids (LI-2I + 25VE, $\mathrm{LI}-2 \mathrm{I}+50 \mathrm{VE}$, respectively); however, no such effect is found 100-fold VE treatment (LI-2I+100VE; G). Scale bar $=20 \mu \mathrm{m}$. 

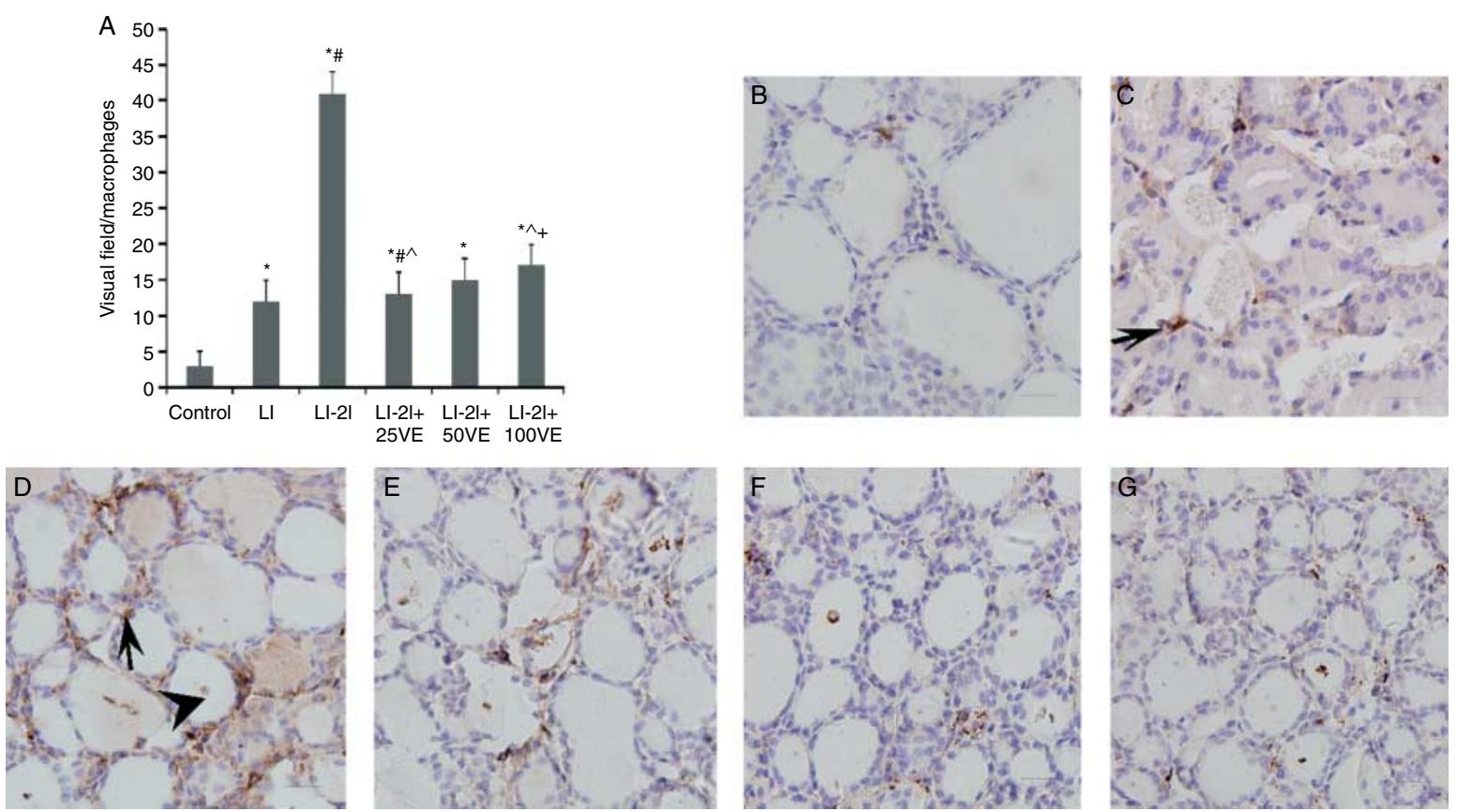

Figure 4 (A) Quantitation of CD68-positive cells. ${ }^{*} P<0 \cdot 01$, compared with control; ${ }^{\sharp} P<0 \cdot 01$, compared with $\mathrm{LI}$; ${ }^{\wedge} P<0 \cdot 01$, compared with LI-2I; ${ }^{+} P<0 \cdot 01$, compared with LI-2I+25VE $(n=8)$. (B-G) Immunohistochemical detection of CD68. (B) In control thyroids, CD68 expression was very low. (C) CD68-positive cells were localized in the interstitium (arrow) and the number was increased in $\mathrm{LI}$-induced goitrous thyroids. (D) In 2I-induced involuting thyroids (LI-2I), CD68-positive cells were significantly increased compared with control (B) and $\mathrm{LI}(\mathrm{C})$, and mostly localized in the interstitium (arrow) as well as in follicular lumina (arrowhead). VE treatment reduced CD68-positive cells in $2 \mathrm{I}$-induced involuting thyroids (LI-2I $+25 \mathrm{VE}, \mathrm{LI}-2 \mathrm{I}+50 \mathrm{VE}$, and $\mathrm{LI}-2 \mathrm{I}$ $+100 \mathrm{VE}$; E-G). Scale bar $=20 \mu \mathrm{m}$.

\section{Antioxidant enzyme expression}

TrxR-1 is an important antioxidant found in the thyroid. It was primarily expressed in the cytoplasm of thyrocytes in control thyroids (Fig. 5B). The staining was less prominent but more diffused throughout the cytoplasm in thyroids of goitrous rats (Fig. 5C). The signal was further suppressed in the thyroids of iodine-induced involuting rats, and this decrease was significant (Fig. 5D). VE treatment restored TrxR-1 expression in the twofold physiological dose of iodine-induced involution groups $(P<0.01$ for $25 \mathrm{VE}$ and 50VE; $P<0.05$ for 100VE versus LI +2 I group; Fig. $5 \mathrm{E}-\mathrm{G})$. The staining was more pronounced and more diffuse throughout the cytoplasm and the staining was also present in some nuclei and interstitium in VE-treated groups.

Another antioxidant, PRDX5, was also weakly expressed in the cytoplasm of control thyrocytes (Fig. $5 \mathrm{~B}^{\prime}$ ). Interestingly, the staining was stronger in goitrous thyroids $(P<0 \cdot 01$ versus control; Fig. $5 \mathrm{C}^{\prime}$ ), where the cytoplasm and numerous nuclei were labeled. Weak expression was observed in the interstitium. In iodine-treated rats, the staining was less pronounced and more diffuse throughout the interstitium as well as in the cytoplasm and some nuclei $\left(\right.$ Fig. $\left.5 \mathrm{D}^{\prime}\right)$. In the 50-fold physiological dose of VE-treated animals (Fig. $5 \mathrm{~F}^{\prime}$ ), the expression of PRDX5 was as weak as that seen in control rats. In the 25- and 100-fold physiological dose of VE-treated rats (Fig. $5 \mathrm{E}^{\prime}$ and $\mathrm{G}^{\prime}$ ), the staining was also weaker than that in the LI group, but significantly greater than that in rats treated with the 50-fold physiological dose of VE. PRDX5 localization was predominantly interstitial and cytosolic with some nuclear staining. The IOD value of PRDX5 was low in control animals. The expression was significantly increased in hyperplastic and iodine-induced involuting glands; although the expression was lower in the latter group. It was decreased in three VE supplementation groups, especially in rats treated with the 50-fold physiological dose of VE (Fig. 5A').

\section{Discussion}

Evidence accumulated over the last several decades indicates that the administration of iodide to goiters provokes thyroid cell necrosis/apoptosis and glandular inflammation (Many et al. 1995, Mutaku et al. 2002, Poncin et al. 2008). Table salt has been iodized throughout China since 1996. Our prospective epidemiological study found that iodine supplementation to a level that is more than adequate in a region where iodine intake was previously mildly deficient may accelerate the development of subclinical hypothyroidism to overt hypothyroidism (Teng et al. 2006). Because of the risks associated with iodine-supplementation to iodine-deficiency 

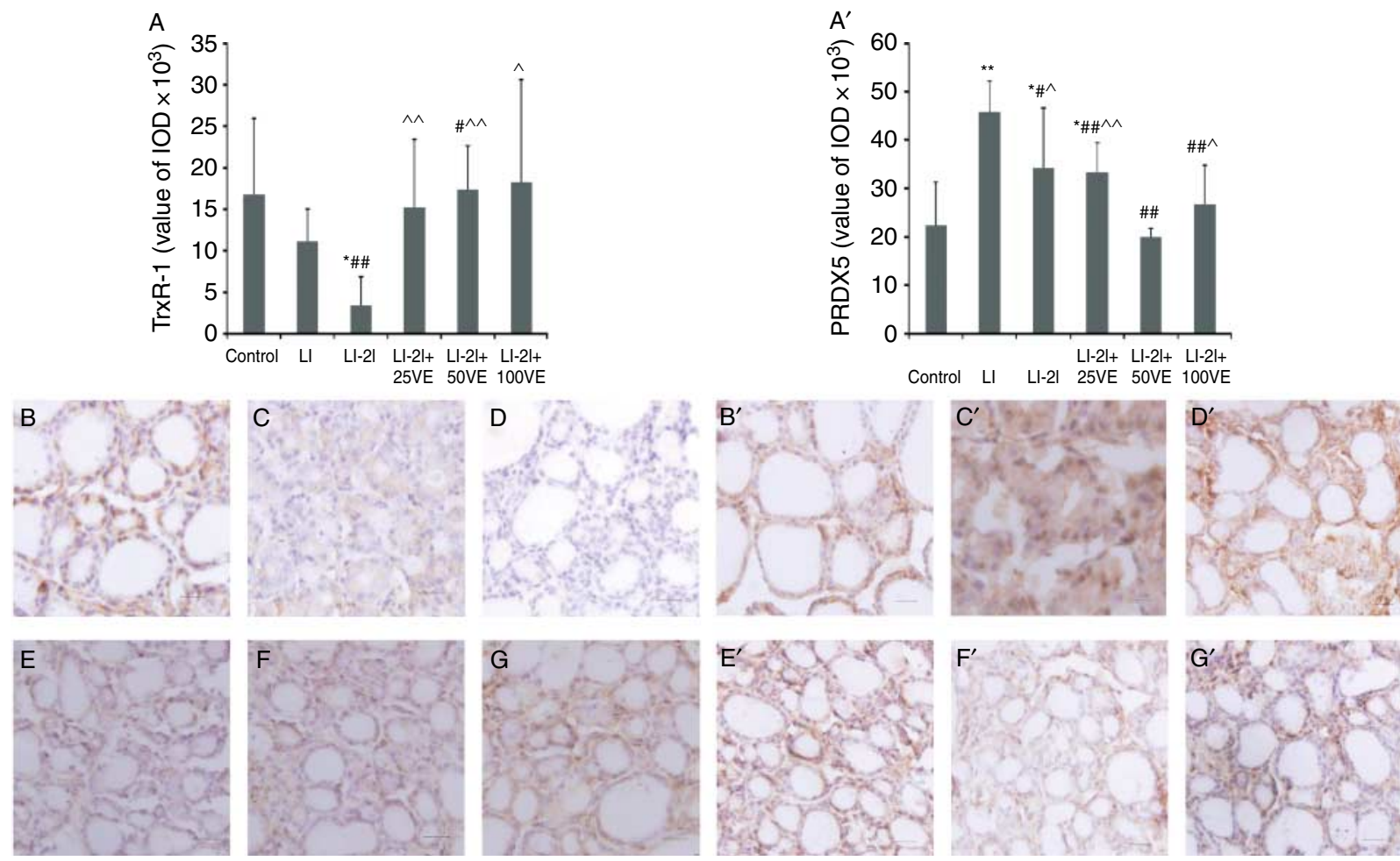

Figure 5 (A) IOD value of TrxR-1. ${ }^{*} P<0 \cdot 01$, compared with control; ${ }^{\sharp} P<0 \cdot 05,{ }^{\# \#} P<0 \cdot 01$, compared with $\mathrm{LI} ;{ }^{\wedge} P<0 \cdot 05,{ }^{\wedge} P<0 \cdot 01$, compared with LI-2I $(n=8)$. (B-G) Immunohistochemical detection of TrxR-1. (B) In control thyroids, TrxR-1 was expressed primarily in the cytoplasm. (C) TrxR-1 was diffused in cytoplasm in LI-induced goitrous thyroids. (D) In 2l-induced involuting thyroids (LI-2I), the staining was less pronounced but was more diffused throughout the cytoplasm. (E-G) VE supplementation significantly restored TrxR-1 expression in $2 \mathrm{I}$-induced involuting thyroids (LI-2I+25VE, LI-2I+50VE, LI-2I + 100VE, respectively). (A') IOD value of PRDX5.

${ }^{*} P<0 \cdot 05,{ }^{* *} P<0 \cdot 01$, compared with control; ${ }^{\sharp} P<0 \cdot 05,{ }^{\sharp} P<0 \cdot 01$, compared with $\mathrm{LI} ;{ }^{\wedge} P<0 \cdot 05,{ }^{\wedge} P<0 \cdot 01$, compared with $\mathrm{LI}-2 \mathrm{I}+$ 50VE $(n=8)$. ( $\left(B^{\prime}-G^{\prime}\right)$ Immunohistochemical detection of PRDX5. ( $\left.B^{\prime}\right)$ PRDX5 was weakly expressed in the cytoplasm in control thyroids. $\left(C^{\prime}\right)$ PRDX5 staining was significantly stronger in $\mathrm{LI}$-induced goitrous thyroids. Not only cytoplasm but also numerous nuclei were labeled and PRDX5 was weakly expressed in the interstitium. ( $\left.{ }^{\prime}\right)$ PRDX5 staining was less pronounced and more diffused throughout the interstitium as well as in cytoplasm and some nuclei in $2 \mathrm{I}$-induced involuting thyroids (LI-2I). ( $\left.\mathrm{E}^{\prime}-\mathrm{G}^{\prime}\right)$ VE supplementation reduced PRDX5 expression in $2 \mathrm{I}$-induced involuting thyroids (LI-2I + 25VE, LI-2I+50VE, and LI-2I+100VE, respectively), especially the LI-2I+ 50VE group. Scale bar $=20 \mu \mathrm{m}$.

goiter and the necessity for iodine supplementation to prevent and treat iodine-deficiency disorders, we need to optimize iodine supplementation and find a way to alleviate or avoid potential iodine-induced thyroid cytotoxicity. VE is an important factor for the cellular protection of membrane lipid peroxidation. In this study, excess iodine (twofold physiological dose) did lead to thyroid cytotoxicity and at least partially alleviated iodine-induced cytotoxicity, possibly through regulating OS, antioxidant defenses, and the inflammatory reaction.

In contrast to previous studies (Poncin et al. 2008, 2010), we observed that OS was decreased in goitrous thyrocytes. The following points may explain this discrepancy. First, antioxidant PRDX5 expression was significantly higher in goitrous glands compared with control, which could play a role in regulating ROS levels in intact thyrocytes. Secondly, Song et al. (2007) have suggested that thyroid peroxidase (TPO) has a catalase-like effect in the absence of iodide; it oxidizes other potential available substrates depending on their specific affinities and thus catabolizes $\mathrm{H}_{2} \mathrm{O}_{2}$. Fang et al. (2001) have demonstrated that TPO mRNA expression was increased in goiters, which is consistent with our previous study that suggested that TPO activity was significantly higher in goiters than in control (A Stone, unpublished observations). Our goitrous gland model was achieved by administering a LI diet without PTU in rats, which suppresses the activity of TPO. This suggests that higher TPO activity may cleave free radicals such as $\mathrm{H}_{2} \mathrm{O}_{2}$ when their levels accumulate in long-term iodine deficiency. Finally, Mutaku et al. (1998) have demonstrated that cell necrosis increased as early as 8 days after goiter induction and returned to control levels by day 32 , which was in line with our findings in the rat goiter model induced for 12 or 16 weeks.

Different from goitrous glands, hyperplastic thyrocytes treated with a twofold physiological dose of iodide were severely injured as shown by the membrane rupture, formation of ER vesicles, mitochondrial swelling, and massive inflammatory response. All of these changes have 
also been found in onefold dose iodine-treated goiter rats in our laboratory (A Stone, unpublished observations). The acuteness of cellular damage was specifically due to iodineinduced toxicity and not to the involution process, which had been supported by $\mathrm{T}_{4}$-induced involution (Poncin et al. 2008). In chronic and twofold dose iodine-treated rats, OS was significantly higher and accompanied by a stronger inflammatory reaction compared with goitrous animals, which is consistent with the findings of Poncin et al. (2008). However, the exact underlying mechanism for excess iodineinduced toxicity in goitrous animals is unclear. Corvilain $e t$ al. (2000) have found that iodide has a stimulatory effect on $\mathrm{H}_{2} \mathrm{O}_{2}$ generation, thereby increasing OS. Iodine-induced cytotoxicity may be due to increased free radicals and more severely altered antioxidant defenses compared with goiter (Poncin et al. 2008). We observed that TrxR-1 and PRDX5 (both are important antioxidants in the thyroid) were expressed at significantly lower levels in iodine-treated rats than goitrous rats, suggesting that these antioxidants may fail to detoxify a heavier load of ROS or that they are depleted by an overload of ROS. Whereas thyroid antioxidant systems are naturally adapted to detoxify excess $\mathrm{H}_{2} \mathrm{O}_{2}$ produced in TSHstimulated thyrocytes, they may be overwhelmed by a heavier and more severe free radical storm-like attack (lipid peroxides, singlet oxygen, superoxide anions, hydroxyl radicals, peroxynitrites, etc.). This hypothesis is in accordance with previous work that demonstrated that tissue damage is markedly enhanced when iodide is supplied to goitrous thyrocytes with impaired antioxidant systems (Contempre et al. 1993, 2004, Mutaku et al. 2002).

Our previous data indicated that selenium may increase antioxidant defenses and partly alleviate iodine-induced thyroid cytotoxicity (A Stone, unpublished observations). In this study, we investigated whether other antioxidants could alleviate iodine-induced cytotoxicity. VE plays a major role in the maintenance of membrane integrity, being both a potent free radical scavenger and a structural stabilizer (Gutteridge 1978). VE deficiency in the thyroid leads to increased malondialdehyde concentration, decreased GPx activity, induction of colloid accumulation and cell debris during goiter involution, and aggravation of epithelial cell necrosis (Mutaku et al. 2002). VE can protect thyrocyte membranes, and it is as concentrated in the thyroid as in the liver in control rats and is increased twofold in goiters (Mutaku et al. 1998). We speculated that the increased concentration of VE in goiter thyroid may compensate for the increased levels of OS. In the 25- and 50-fold physiological dose VE-treated groups, the expressions of 4-HNE and 8-OHdG were significantly decreased. The 100-fold physiological dose of VE decreased the expression of 8-OHdG, but 4-HNE remained significantly higher than that observed in the iodine-treated group. Although VE is an antioxidant, overdose may increase OS (Bouayed \& Bohn 2010). VE alleviated the over-activated OS induced by iodine. The expression of TrxR-1 was significantly higher in all three VE supplementation groups compared with the iodine-treated group, which suggests that VE may alleviate ROS by upregulating TrxR-1. However, PRDX5 expression was decreased in all three VE supplementation groups, especially in the 50-fold group $(P<0 \cdot 01)$, compared with LI group. Previous studies indicated that the administration of $\mathrm{N}$-acetylcysteine, a potent antioxidant, reduces PRDX5 expression in the thyroid (Poncin et al. 2010), therefore we presume that VE may act in the same manner.

In conclusion, long-term supplementation using a twofold dose of iodine may induce iodine-deficient thyroid involution, and lead to iodine-induced thyroid cytotoxicity. Excessive OS may play an important role in the pathophysiology of iodine-induced thyroid toxicity. VE may increase thyroid antioxidant defenses and inhibit iodine-induced thyroid cytotoxicity. Further studies are needed to verify the optimal dose of VE supplementation for ameliorating the potential side effects of universal salt iodization.

\section{Declaration of interest}

The authors declare that there is no conflict of interest that could be perceived as prejudicing the impartiality of the research reported.

\section{Funding}

This work was supported by China Guanghua Foundation (Grant number [2007]02) and the Science and Technology Program of Liaoning Province (Grant number 2010225019).

\section{Acknowledgements}

The authors would like to thank Prof. Zhang Fuhui (Laboratory of Electron Microscopy, China Medical University) for his continual support.

\section{References}

Abd-El-Fattah A, El-Sawalhi MM, Rashed ER \& El-Ghazaly MA 2010 Possible role of vitamin E, coenzyme Q10 and rutin in protection against cerebral ischemia/reperfusion injury in irradiated rats. International Journal of Radiation Biology 86 1070-1078. (doi:10.3109/09553002.2010.501844)

Banmeyer I, Marchand C, Clippe A \& Knoops B 2005 Human mitochondrial peroxiredoxin 5 protects from mitochondrial DNA damages induced by hydrogen peroxide. FEBS Letters 579 2327-2333. (doi:10.1016/j.febslet. 2005.03.027)

Bouayed J \& Bohn T 2010 Exogenous antioxidants - double-edged swords in cellular redox state: health beneficial effects at physiologic doses versus deleterious effects at high doses. Oxidative Medicine and Cellular Longevity 3 228-237. (doi:10.4161/oxim.3.4.12858)

Chong W, Chen W, Teng WP, Shan ZY, Jin Y, Zhang XP, Xi LM, Zhang N, Man N \& Tong YJ 2005 Establish and evaluation of iodine deficient animal model. Journal of China Medical University 34 111-113.

Chong W, Chen W, Shan ZY, Jin Y, Guan HX, Li YS, Zhang N, Man N, Tong YJ \& Teng WP 2006a Effect of chronic excessive iodine on thyroid morphology of iodine-deficient rats. China Journal of Modern Medicine 16 1781-1785.

Chong W, Teng D, Shi XG, Shan ZY \& Teng WP $2006 b$ Effect of iodine supplement on thyroid of iodine-deficient rats. Chinese Journal of Practical Internal Medicine 26 0583-0585. 
Contempre B, Denef JF, Dumont JE \& Many MC 1993 Selenium deficiency aggravates the necrotizing effects of a high iodide dose in iodine deficient rats. Endocrinology 132 1866-1868. (doi:10.1210/en.132.4.1866)

Contempre B, de Escobar GM, Denef JF, Dumont JE \& Many MC 2004 Thiocyanate induces cell necrosis and fibrosis in selenium- and iodinedeficient rat thyroids: a potential experimental model for myxedematous endemic cretinism in central Africa. Endocrinology 145 994-1002. (doi:10.1210/en.2003-0886)

Corvilain B, Collyn L, Van SJ \& Dumont JE 2000 Stimulation by iodide of $\mathrm{H}_{2} \mathrm{O}_{2}$ generation in thyroid slices from several species. American Journal of Physiology. Endocrinology and Metabolism 278 E692-E699.

Denef JF, Many MC \& van den Hove MF 1996 Iodine-induced thyroid inhibition and cell necrosis: two consequences of the same free-radical mediated mechanism? Molecular and Cellular Endocrinology 121 101-103. (doi:10.1016/0303-7207(96)03848-8)

Dubuisson M, Vander SD, Clippe A, Etienne F, Nauser T, Kissner R, Koppenol WH, Rees JF \& Knoops B 2004 Human peroxiredoxin 5 is a peroxynitrite reductase. FEBS Letters 571 161-165. (doi:10.1016/j.febslet. 2004.06.080)

Fang H, Yan YQ, Chen ZP \& Xu G 2001 Thyroid function and expressions of thyroglobulin and thyroid peroxidase mRNA in rats at different iodine intake levels. Chinese Journal of Endocrinology and Metabolism 17 83-85.

Fibach E \& Rachmilewitz EA 2010 The role of antioxidants and iron chelators in the treatment of oxidative stress in thalassemia. Annals of the New York Academy of Sciences 1202 10-16. (doi:10.1111/j.1749-6632.2010.05577.x)

Follis RH Jr 1959 Thyroiditis resulting from administration of excess iodine to hamsters with hyperplastic goiters. Proceedings of the Society for Experimental Biology and Medicine 102 425-429.

Gerard AC, Many MC, Daumerie C, Knoops B \& Colin IM 2005 Peroxiredoxin 5 expression in the human thyroid gland. Thyroid $\mathbf{1 5}$ 205-209. (doi:10.1089/thy.2005.15.205)

Gutteridge JMC 1978 The membrane effects of vitamin E, cholesterol and their acetates on peroxidative susceptibility. Research Communications in Chemical Pathology and Pharmacology 22 563-572.

Kohrle J, Jakob F, Contempre B \& Dumont JE 2005 Selenium, the thyroid, and the endocrine system. Endocrine Reviews 26 944-984. (doi:10.1210/er. 2001-0034)

Lillig CH \& Holmgren A 2007 Thioredoxin and related molecules - from biology to health and disease. Antioxidants and Redox Signaling $92-47$.

Many MC, Papadopoulous J, Martin C, Colin I \& Denef JF 1991 Iodine induced cell damage in mouse hyperplastic thyroid is associated to lipid peroxidation. In Progress in Thyroid Research, pp 635-638. Eds A Gordon, J Gross \& G Henemann. Rotterdam: Balkema.

Many MC, Maniratunga S, Varis I, Dardenne M, Drexhage HA \& Denef JF 1995 Two-step development of Hashimoto-like thyroiditis in genetically autoimmune prone non-obese diabetic mice: effects of iodine-induced cell necrosis. Journal of Endocrinology 147 311-320. (doi:10.1677/joe.0.1470311)

Martino E, Safran M, Aghini-Lombardi F, Rajatanavin R, Lenziardi M, Fay M, Pacchiarotti A, Aronin A, Macchia E \& Haffajee C 1984 Environmental iodine intake and thyroid dysfunction during chronic amiodarone therapy. Annals of Internal Medicine 101 28-34.

Mikami T, Ichiseki T, Kaneuji A, Ueda Y, Sugimori T, Fukui K \& Matsumoto T 2010 Prevention of steroid-induced osteonecrosis by intravenous administration of vitamin $\mathrm{E}$ in a rabbit model. Journal of Orthopaedic Science 15 674-677. (doi:10.1007/s00776-010-1516-7)
Milman U, Blum S, Shapira C, Aronson D, Miller-Lotan R, Anbinder Y, Alshiek J, Bennett L, Kostenko M \& Landau M 2008 Vitamin E supplementation reduces cardiovascular events in a subgroup of middleaged individuals with both type 2 diabetes mellitus and the haptoglobin 2-2 genotype: a prospective double-blinded clinical trial. Arteriosclerosis, Thrombosis, and Vascular Biology 28 341-347. (doi:10.1161/ATVBAHA.107. 153965)

Mutaku JF, Many MC, Colin I, Denef JF \& van den Hove MF 1998 Antigoitrogenic effect of combined supplementation with dl-alphatocopherol, ascorbic acid and beta-carotene and of dl-alpha-tocopherol alone in the rat. Journal of Endocrinology 156 551-561. (doi:10.1677/joe.0. 1560551)

Mutaku JF, Poma JF, Many MC, Denef JF \& van den Hove MF 2002 Cell necrosis and apoptosis are differentially regulated during goiter development and iodine-induced involution. Journal of Endocrinology 172 375-386. (doi:10.1677/joe.0.1720375)

Poncin S, Gerard AC, Boucquey M, Senou M, Calderon PB, Knoops B, Lengele B, Many MC \& Colin IM 2008 Oxidative stress in the thyroid gland: from harmlessness to hazard depending on the iodine content. Endocrinology 149 424-433. (doi:10.1210/en.2007-0951)

Poncin S, Van Eeckoudt S, Humblet K, Colin IM \& Gérard AC 2010 Oxidative stress: a required condition for thyroid cell proliferation. American Journal of Pathology 176 1355-1363. (doi:10.2353/ajpath.2010. 090682)

Saxena S, Srivastava P \& Khanna VK 2010 Antioxidant supplementation improves platelet membrane fluidity in idiopathic retinal periphlebitis (Eales' disease). Journal of Ocular Pharmacology and Therapeutics 26 623-626. (doi:10.1089/jop.2010.0075)

Schweizer U, Chiu J \& Köhrle J 2008 Peroxides and peroxide-degrading enzymes in the thyroid. Antioxidants and Redox Signaling 10 1577-1592. (doi:10.1089/ars.2008.2054)

Song Y, Driessens N, Costa M, De Deken X, Detours V, Corvilain B, Maenhaut C, Miot F, Van SJ, Many MC et al. 2007 Roles of hydrogen peroxide in thyroid physiology and disease. Journal of Clinical Endocrinology and Metabolism 92 3764-3773. (doi:10.1210/jc.2007-0660)

Stanbury JB, Ermans AE, Bourdoux P, Todd C, Oken E, Tonglet R, Vidor G, Braverman LE \& Medeiros-Neto G 1998 Iodine-induced hyperthyroidism: occurrence and epidemiology. Thyroid 8 83-100. (doi:10.1089/thy. 1998.8.83)

Teng W, Shan Z, Teng X, Guan H, Li Y, Teng D, Jin Y, Yu X, Fan C \& Chong W 2006 Effect of iodine intake on thyroid diseases in China. New England Journal of Medicine 354 2783-2793. (doi:10.1056/NEJMoa054022)

Zhang N, Tong YJ, Shan ZY \& Teng WP 2006 Effect of chronic mild and moderate iodine excess on thyroid anti-oxidative ability of iodine deficiency and non-iodine deficiency Wistar rats. National Medical Journal of China 86 1274-1277.

Received in final form 3 March 2011

Accepted 14 March 2011

Made available online as an Accepted Preprint 15 March 2011 